\title{
Bi-Lipschitz decomposition of Lipschitz functions into a metric space
}

Raanan Schul

\begin{abstract}
We prove a quantitative version of the following statement. Given a Lipschitz function $f$ from the k-dimensional unit cube into a general metric space, one can be decomposed $f$ into a finite number of BiLipschitz functions $\left.f\right|_{F_{i}}$ so that the k-Hausdorff content of $f\left([0,1]^{k} \backslash \cup F_{i}\right)$ is small. We thus generalize a theorem of $\mathrm{P}$. Jones [7] from the setting of $\mathbb{R}^{d}$ to the setting of a general metric space. This positively answers problem 11.13 in "Fractured Fractals and Broken Dreams" by G. David and S. Semmes, or equivalently, question 9 from "Thirtythree yes or no questions about mappings, measures, and metrics" by J. Heinonen and S. Semmes. Our statements extend to the case of coarse Lipschitz functions.
\end{abstract}

\section{Introduction}

We prove the following theorem.

Theorem 1.1. Let $\epsilon \geq 0,0<\alpha<1$ and $k \geq 1$ be given. There are universal constants $M=M(\alpha, k), c_{1}=c_{1}(k)$ and $c_{2}$ such that the following statements hold. Let $\mathcal{M}$ be any metric space. Let $f:[0,1]^{k} \rightarrow \mathcal{M}$ be an $\epsilon$-coarse 1-Lipschitz function, i.e. such that

$$
\operatorname{dist}(f(x), f(y)) \leq|x-y|+\epsilon
$$

Then there are sets $F_{1}, \ldots, F_{M} \subset[0,1]^{k}$ so that for $1 \leq i \leq M, x, y \in F_{i}$ we have

$$
\alpha|x-y|-c_{2} \epsilon \leq \operatorname{dist}(f(x), f(y)) \leq|x-y|+\epsilon,
$$

2000 Mathematics Subject Classification: Primary: 28A75; Secondary: 42C99, 51F99. Keywords: Lipschitz, Bi-Lipschitz, metric space, uniform rectifiability, Sard's theorem. 
and

$$
h^{k}\left(f\left([0,1]^{k} \backslash\left(F_{1} \cup \ldots \cup F_{M}\right)\right)\right) \leq c_{1} \alpha .
$$

( $h^{k}$ is the one-dimensional Hausdorff content, defined below).

As a corollary of the theorem (with $\epsilon=0$ ) we get

Corollary 1.2. Let $k>0$. Let $\mathcal{M}$ be a $k$-Ahlfors-David regular metric space which has Big Pieces of Lipschitz images of $\mathbb{R}^{k}$. Then $\mathcal{M}$ has Big Pieces of BiLipshcitz images of $\mathbb{R}^{k}$.

Thus we positively answer problem 11.13 in [4] by G. David and S. Semmes, or equivalently, question 9 in [6] by J. Heinonen and S. Semmes.

The statement of Corollary 1.2 for the case where $\mathcal{M}=\mathbb{R}^{d}$ was proved by Guy David [1]. In fact, David assumes less about the domain of the functions in question. In particular, the domains need not be Euclidean, however they are required to satisfy some geometric conditions. Shortly after, Peter Jones gave a proof for Theorem 1.1 for the case where $\{\epsilon=0$ and $\left.\mathcal{M}=\mathbb{R}^{d}\right\}$ [7]. (Jones' and David's results appeared in the same issue of Rev. Mat. Iberoamericana.) Their work was motivated by the study of singular integrals.

Theorem 1.1, with $\epsilon=0$, can be thought of as a quantitative version of Sard's Theorem, where we think of the non-quantitative version as: a Lipschitz map can be written as a countable union of invertible maps, and a map whose range is a Lebesgue null set. We note that a non-quantitative variant of our theorem had already appeared in [8].

The proof we give follows the outline of [7]. An important point is that the $\mathbb{R}^{d}$ result relies on a sum of squares of wavelet coefficients (see the exposition in [2]) or their upper half space analogue (which was the way the proof went in [7]). We replace this by a statement about a metric space analogue of the Jones $\beta$ numbers, or equivalently, a statement about certain Menger curvature averages (Lemma 2.1). It is the authors feeling that the lack of such a statement was the only thing that prevented this theorem from appearing 10-20 years ago. This theorem is another building block in the process of transferring (parts of) the Euclidean theory of quantitative rectifiability to the setting of general metric spaces.

Let us quickly define the relevant notions. A metric space $\mathcal{M}$ is said to be a $k$-Ahlfors-David regular (with constant $c_{1}$ ) if for any $x \in \mathcal{M}, 0<$ $r<\operatorname{diam}(\mathcal{M})$ we have $c_{1}^{-1} r^{k} \leq \mathcal{H}^{k}(\operatorname{Ball}(x, r)) \leq c_{1} r^{k}$. A $k$-Ahlfors-David regular metric space $\mathcal{M}$ is said to have Big Pieces of Lipschitz images (with constants $L_{1}$ and $c_{1}$ ) if for any $x \in \mathcal{M}, 0<r<\operatorname{diam}(\mathcal{M})$ we have 
an $L_{1}$ Lipschitz function $f: A \rightarrow \mathcal{M}$, where $A \subset \operatorname{Ball}_{\mathbb{R}^{k}}(0, r)$ such that $\mathcal{H}^{k}(f(A) \cap \operatorname{Ball}(x, r)) \geq c_{1} r^{k}$. A $k$-Ahlfors-David regular metric space $\mathcal{M}$ is said to have Big Pieces of BiLipschitz images (with constants $L_{2}$ and $c_{2}$ ) if for any $x \in \mathcal{M}, \quad 0<r<\operatorname{diam}(\mathcal{M})$ we have an $L_{2}$ BiLipschitz function $f: A \rightarrow \mathcal{M}$, where $A \subset \operatorname{Ball}_{\mathbb{R}^{k}}(0, r)$ such that $\mathcal{H}^{k}(f(A) \cap \operatorname{Ball}(x, r)) \geq c_{2} r^{k}$.

See [3] or [4] for more details. We note that in question 9 of [6] there is an error in the definition of Big Pieces of Lipschitz images.

Proof of Corollary 1.2. Let $c_{1}, x, r, L_{1} f, A$ as in the definition of Big Pieces of Lipschitz images be given. Let $e: \mathcal{M} \rightarrow L^{\infty}(\mathcal{M})$ be the Kuratowski embedding. Using the McShane-Whitney extension lemma for each coordinate, we extend $e \circ f$ to a $L_{1}$-Lipschitz function $\tilde{f}:[-r, r]^{k} \rightarrow$ $L^{\infty}(M)$. See page 10 in [5] for more details. We now apply Theorem 1.1 (rescaled) to $\tilde{f}:[-r, r]^{k} \rightarrow L^{\infty}(M)$ with sufficiently small $\alpha$ (depending on the $k$-Ahlfors-David-regularity constant of $\mathcal{M}$, as well as $L_{1}$, and $k$ ) and $\epsilon=0$ to get $\mathcal{H}^{k}\left(f\left(E \backslash\left(\cup F_{i}\right)\right)\right) \leq \frac{1}{2} c_{1} r^{k}$. Hence one of the sets $E \cap F_{i}$ must satisfy $\mathcal{H}^{k}\left(f\left(E \cap F_{i}\right) \cap \operatorname{Ball}(x, r)\right) \geq \frac{c_{1}}{2 M} r^{k}$, as desired.

Acknowledgments. The author would like to thank Stephen Keith for pointing out a mistake in an earlier version of this essay. We also thank the anonymous referee for pointing out a significant oversight in the discussion of history which follows Corollary 1.2.

\section{Proof of Theorem 1.1}

\subsection{Definitions}

For a set $E$, define the one-dimensional Hausdorff content of a set $K$ as

$$
h^{k}(E)=\inf \left\{\sum \operatorname{diam}\left(U_{i}\right)^{k}: \cup U_{i} \supset E\right\} .
$$

Let $p$ be a function with range contained in $\mathcal{M}$. Define

$$
\partial_{1}(x, y, z)=\partial_{1}^{(p)}(x, y, z)
$$

by

$$
\partial_{1}(x, y, z)=\operatorname{dist}(p(x), p(y))+\operatorname{dist}(p(y), p(z))-\operatorname{dist}(p(x), p(z)) .
$$

Define for an interval $I=[a, b] \subset \mathbb{R}$ the quantity $\tilde{\beta}(I)=\tilde{\beta}_{(p)}(I)$ by

$$
\tilde{\beta}(I)^{2} \operatorname{diam}(I)=\operatorname{diam}(I)^{-3} \int_{x=a}^{x=b} \int_{y=x}^{y=b} \int_{z=y}^{z=b} \partial_{1}(x, y, z) d z d y d x .
$$

We extend this definition to higher dimensional cubes by rotations. 
Let $k>1$. Define for a cube $Q \in \mathbb{R}^{k}$ the quantity $\tilde{\beta}^{(k)}(Q)=\tilde{\beta}_{(p)}^{(k)}(Q)$ by

$$
\begin{aligned}
& \tilde{\beta}^{(k)}(Q)^{2} \operatorname{side}(Q)^{k-1}= \\
& \quad=\int_{g \in G_{k}} \int_{x \in \mathbb{R}^{k} \ominus g \mathbb{R}} \chi_{\{|(x+g \mathbb{R}) \cap 7 Q| \geq \operatorname{side}(Q)\}} \tilde{\beta}((x+g \mathbb{R}) \cap 7 Q)^{2} d x d \mu(g)
\end{aligned}
$$

where $\mathbb{R}$ is identified with $\{\mathbb{R}, 0, \ldots, 0\} \subset \mathbb{R}^{k}, G_{k}$ is the group of all rotations of $\mathbb{R}$ in $\mathbb{R}^{k}$ equipped with the its Haar measure $d \mu$, and $d x$ is the $k-1$ dimensional Lebesgue measure on $\mathbb{R}^{k} \ominus g \mathbb{R}$, the orthogonal complement of $g \mathbb{R}$ in $\mathbb{R}^{k}$. We write $\tilde{\beta}^{(1)}=\tilde{\beta}$. and note that any $k \geq 1$, we have that $\tilde{\beta}^{(k)}$ is scale invariant. This type of quantity is connected to Menger curvature. See [10] for more details.

Define $\mathcal{D}_{0}$ the standard dyadic partition of $\mathbb{R}$, i.e.

$$
\mathcal{D}_{0}:=\left\{\left[\frac{j_{1}}{2^{j_{2}}}, \frac{j_{1}+1}{2^{j_{2}}}\right]: j_{1}, j_{2} \in \mathbb{Z}\right\} .
$$

Define $\mathcal{D}_{1}$ a dyadic partition of $\mathbb{R}$ given by shifting the standard dyadic partition by $\frac{1}{3}$, i.e. $\mathcal{D}_{0}+\frac{1}{3}$. For $i=\left(i_{1}, \ldots, i_{k}\right) \in\{0,1\}^{k}$ we define

$$
\mathcal{D}_{i}^{k}:=\mathcal{D}_{i_{1}} \oplus \cdots \oplus \mathcal{D}_{i_{k}} \text {. }
$$

The fact that now a ball $\operatorname{Ball}(x, r) \subset \mathbb{R}^{k}$ with $r<\frac{1}{6}$ is contained in a cube $Q \in \cup_{i \in\{0,1\}^{k}} \mathcal{D}_{i}^{k}$ with $\operatorname{side}(Q) \sim r$ earns this setup the (now standard) name the one third trick.

For simplicity of notation in the proof, we extend $f$ to be 1-Lipschitz with domain $\mathbb{R}^{k}$ (by say fixing $f$ on rays emanating from $\left(\frac{1}{2}, \ldots, \frac{1}{2}\right)$ and outside $(0,1)^{k}$.

We call two dyadic cubes $Q_{1}$ and $Q_{2}$ semi-adjacent if

$$
0<\operatorname{dist}\left(Q_{1}, Q_{2}\right) \leq 2 \operatorname{diam}\left(Q_{1}\right)=2 \operatorname{diam}\left(Q_{2}\right) .
$$

Hence every cube $Q$ has at most $C(k)$ semi-adjacent cubes.

\subsection{The proof}

We start by defining a Lipschitz function $p$.

Let $X=X_{\epsilon} \subset[0,1]^{k}$ be an $\epsilon$-net for $[0,1]^{k}$ and $Z=Z_{\epsilon} \subset \mathcal{M}$ be an $\epsilon$-net for $\mathcal{M}$. Consider a function $f^{\prime}: X \rightarrow \mathcal{M}$ defined as follows. for $z \in \mathcal{M}$, let $z_{\epsilon} \in Z$ be such that $\operatorname{dist}\left(z, z_{\epsilon}\right) \leq \epsilon$ (chosen arbitrarily if there is more then one such $\left.z_{\epsilon}\right)$. Define $f^{\prime}(x)=f(x)_{\epsilon}$. We have for any $x, y \in X$

$$
\operatorname{dist}\left(f^{\prime}(x), f(x)\right) \leq \epsilon, \quad \operatorname{dist}\left(f^{\prime}(x), f^{\prime}(y)\right) \leq|x-y|+\epsilon+2 \epsilon,
$$

We get that for any $x, y \in X$

$$
\operatorname{dist}\left(f^{\prime}(x), f^{\prime}(y)\right) \leq 4|x-y| .
$$


Now extend $f^{\prime}$ to a 4-Lipschitz function $p:[0,1]^{k} \rightarrow L^{\infty}(\mathcal{M})$ using the Kuratowski embedding and the McShane-Whitney extension (as in Corollary 1.2). Denote by $\tilde{f}:[0,1]^{k} \rightarrow L^{\infty}(\mathcal{M})$, the map given by using the Kuratowski embedding of $\mathcal{M}$ in $L^{\infty}(\mathcal{M})$. We have for $x \in[0,1]^{k}$ and $x_{\epsilon} \in X$ such that $\operatorname{dist}\left(x, x_{\epsilon}\right) \leq \epsilon$

$$
\operatorname{dist}(\tilde{f}(x), p(x)) \leq \operatorname{dist}\left(\tilde{f}(x), \tilde{f}\left(x_{\epsilon}\right)\right)+\operatorname{dist}\left(\tilde{f}\left(x_{\epsilon}\right), p\left(x_{\epsilon}\right)\right)+\operatorname{dist}\left(p\left(x_{\epsilon}\right), p(x)\right)
$$

$$
\leq 2 \epsilon+\epsilon+4 \epsilon \text {. }
$$

This $p$ is the one we use in the above definitions of $\partial_{1}$ and $\beta$.

Lemma 2.1. For an L-Lipschitz function $p$,

$$
\sum_{\substack{Q \in \mathcal{D}_{i}^{k}, Q \subset[0,1]^{k} \\ i \in\{0,1\}^{k}}} \tilde{\beta}^{(k)}(Q)^{2} \operatorname{side}(Q)^{k} \lesssim L .
$$

We postpone the proof of this lemma to Section 3.

Let $\alpha^{\prime}=10 \alpha$. For $x_{1}, x_{2} \in \mathbb{R}^{k}$, let $\left[x_{1}, x_{2}\right]$ be the straight segment connecting $x_{1}$ and $x_{2}$. Let

$$
\begin{aligned}
& \mathcal{E}_{1}:=\left\{Q_{1} \in \mathcal{D}_{\overline{0}}^{k}: \quad\right. Q_{1} \subset[0,1]^{k}, \quad \exists x_{1} \in Q_{1}, x_{2} \in Q_{2}, \\
& Q_{1}, Q_{2} \text { semi-adjacent, } \operatorname{diam}\left(p\left(\left[x_{1}, x_{2}\right]\right)\right) \geq \alpha^{\prime}\left|x_{1}-x_{2}\right|, \\
&\left.\operatorname{dist}\left(p\left(x_{1}\right), p\left(x_{2}\right)\right) \leq \frac{\alpha^{\prime}}{10}\left|x_{1}-x_{2}\right|, \quad \alpha^{\prime}\left|x_{1}-x_{2}\right| \geq 10 \epsilon\right\}, \\
& \mathcal{E}_{2}:=\left\{\left[x_{1}, x_{2}\right]: \quad\right. x_{i} \in Q_{i}, \quad Q_{1} \subset[0,1]^{k}, \\
& Q_{1}, Q_{2} \text { semi-adjacent, } \operatorname{diam}\left(p\left(\left[x_{1}, x_{2}\right]\right)\right) \leq \alpha^{\prime}\left|x_{1}-x_{2}\right|, \\
&\left.\alpha^{\prime}\left|x_{1}-x_{2}\right| \geq 10 \epsilon\right\} . \\
& B:=\left\{Q_{0} \in \mathcal{E}_{1}: \quad \exists Q_{1}, \ldots, Q_{N} \in \mathcal{E}_{1}, \text { such that } Q_{0} \subsetneq Q_{1} \subsetneq \ldots \subsetneq Q_{N}\right\} .
\end{aligned}
$$

The constant $N$ will be chosen later, and will depend only on $\alpha^{\prime}$ and $k$. (Note that in the definition of $B$ the cubes $Q_{1}, \ldots, Q_{N}$ may be of wildly different scales.)

\section{Lemma 2.2.}

$$
h^{k}\left(f\left(\cup \mathcal{E}_{2}\right)\right) \lesssim \alpha^{\prime}
$$

Proof. Assume $\left[x_{1}, x_{2}\right] \in \mathcal{E}_{2}$, and let $Q_{1}, Q_{2}$ be the corresponding semiadjacent cubes. Recall that $\left|x_{1}-x_{2}\right| \sim \operatorname{diam}\left(Q_{1}\right)$. Define for $c=3,30$

$$
U_{x_{1}, x_{2}}^{c}=f^{-1} \operatorname{Ball}\left(f\left(x_{1}\right), c \alpha^{\prime}\left|x_{1}-x_{2}\right|\right) .
$$


Then

$$
U_{x_{1}, x_{2}}^{3} \supset\left\{x \in \mathbb{R}^{k}: \operatorname{dist}\left(x,\left[x_{1}, x_{2}\right]\right)<\alpha^{\prime}\left|x_{1}-x_{2}\right|\right\}
$$

Note that

$$
\operatorname{diam}\left(f U_{x_{1}, x_{2}}^{30}\right) \lesssim \alpha^{\prime}\left|x_{2}-x_{1}\right|
$$

implying that (together with (2.2))

$$
\operatorname{diam}\left(f U_{x_{1}, x_{2}}^{30}\right)^{k} \lesssim \alpha^{\prime} \mathcal{H}^{k}\left(U_{x_{1}, x_{2}}^{3}\right)
$$

Consider the set

$$
\mathcal{U}=\left\{U_{x_{1}, x_{2}}^{3}:\left[x_{1}, x_{2}\right] \in \mathcal{E}_{2}\right\} .
$$

We will show $h^{k}(\cup f \mathcal{U}) \lesssim \alpha^{\prime}$, which will give the lemma as $\cup \mathcal{E}_{2} \subset \cup \mathcal{U}$.

We use a Vitali covering type argument. We find a disjoint sub-collection $\mathcal{U}^{\prime} \subset \mathcal{U}$ so that if $U \in \mathcal{U}$ then $U \cap U^{\prime} \neq \emptyset$ for some $U^{\prime} \in \mathcal{U}^{\prime}$ with $2 \operatorname{diam}\left(f U^{\prime}\right) \geq$ $\operatorname{diam}(f U)$ as follows. Write $\mathcal{U}=\cup \mathcal{U}_{j}$ where $U \in \mathcal{U}_{j}$ implies

$$
2^{-j-1}<\operatorname{diam}(f U) \leq 2^{-j} .
$$

We greedily construct $\mathcal{U}^{\prime}$ by adding sets to it from $\mathcal{U}_{j}$, inducting on $j$. We start with $\mathcal{U}^{\prime}=\emptyset$. Place a maximal (with respect to inclusion) disjoint subset of $\mathcal{U}_{0}$ in $\mathcal{U}^{\prime}$. At stage $j>0$, consider all sets $S_{j} \subset \mathcal{U}_{j}$ which have disjoint elements and have elements disjoint from all current $\mathcal{U}^{\prime}$ elements. Take a maximal (with respect to inclusion) such $S_{j}$, and add $S_{j}$ to $\mathcal{U}^{\prime}$. This defines $\mathcal{U}^{\prime}$ as desired.

Now, we note that if $U \in \mathcal{U}$ and $U \cap U^{\prime} \neq \emptyset, U^{\prime} \in \mathcal{U}^{\prime}$ with $2 \operatorname{diam}\left(f U^{\prime}\right) \geq$ $\operatorname{diam}(f U)$, and $U^{\prime}=U_{x_{1}, x_{2}}^{3}$, then (by looking at the push-forward by $f$ ) $U \subset U_{x_{1}, x_{2}}^{30}$. Hence

$$
\cup\{U: U \in \mathcal{U}\} \subset \cup\left\{U_{x_{1}, x_{2}}^{30}: U_{x_{1}, x_{2}}^{3} \in \mathcal{U}^{\prime}\right\} .
$$

Using the disjointness of elements in $\mathcal{U}^{\prime}$ and inequality (2.3) we get the desired result.

Lemma 2.3. There is an $\epsilon_{0}=\epsilon_{0}\left(\alpha^{\prime}, k\right)>0$ such that for any $Q_{1} \in \mathcal{E}_{1}$ we have $\tilde{\beta}^{(k)}\left(7 Q_{1}\right) \geq \epsilon_{0}$.

Proof. Let $Q_{2}, x_{1}, x_{2}$ be as in the definition of $\mathcal{E}_{1}$. Set

$$
D=\operatorname{side}\left(Q_{1}\right) \geq\left|x_{2}-x_{1}\right| .
$$

Let $x_{3} \in\left[x_{1}, x_{2}\right]$ be a point such that $\operatorname{dist}\left(p\left(x_{3}\right), p\left(x_{1}\right)\right)>\frac{\alpha^{\prime}}{2} D$. Then for $x_{i}^{\prime} \in \operatorname{Ball}\left(x_{i}, \frac{\alpha^{\prime}}{100} D\right)$ we have $\partial_{1}\left(x_{1}^{\prime}, x_{3}^{\prime}, x_{2}^{\prime}\right) \geq \frac{\alpha^{\prime}}{10} D$. Now use definition of $\tilde{\beta}^{(k)}$. 
Let

$$
B_{1}=\bigcup_{\substack{Q \in B \\ Q \subset[0,1]^{k}}} 7 Q
$$

From Lemma 2.1, Lemma 2.3, and the one-third trick, we have:

Lemma 2.4. By taking $N=N\left(\alpha^{\prime}, k\right)$ large enough (universally determined)

$$
\mathcal{H}^{k}\left(B_{1}\right) \lesssim \alpha^{\prime}
$$

and

$$
h^{k}\left(f\left(B_{1}\right)\right) \lesssim \alpha^{\prime}
$$

Proof. The first inequality follows from

$$
\int \chi_{B_{1}} \leq \int \frac{1}{N+1} \sum_{Q \in \mathcal{E}_{1}} \chi_{7 Q} \leq \frac{C}{N+1} .
$$

If $\epsilon=0$ this is more then enough for the second inequality as well. If $\epsilon>0$, to see the second inequality we note that

$$
\begin{aligned}
h^{k}\left(f\left([0,1]^{k} \cap B_{1}\right)\right) & \leq \frac{1}{N+1} \sum_{Q \in \mathcal{E}_{1}} \operatorname{side}(7 Q)+\epsilon \\
& \leq \frac{1}{N+1} \sum_{Q \in \mathcal{E}_{1}} 2 \operatorname{side}(7 Q) \leq \frac{C}{N+1} .
\end{aligned}
$$

Now denote by $G=[0,1]^{k} \backslash\left(B_{1} \cup\left(\cup \mathcal{E}_{2}\right)\right)$. We would like to split $G$ into $M\left(\alpha^{\prime}, k\right)$ sets as desired. We split according to the behavior of the function $p$ using $\mathcal{E}_{1}$ as our guide. One goes through the dyadic tree (large scale to fine scale) and makes sure that if $Q_{1}, Q_{2} \in \mathcal{E}_{1}$ are semi-adjacent, then they are not in the same $F_{i}$. Since we excise the intervals in $B$ (which gave us $B_{1}$ ), we can do this with only a finite number of sets $F_{i}$ (namely $M=2^{C(k) N}$ ). For more details see pages 81-82 of [2] (starting at the bottom of page 81, with the same notation). This gives $F_{1}, \ldots, F_{M} \subset[0,1]^{k}$ so that for $1 \leq i \leq M$, and $x, y \in F_{i}$ such that $\alpha^{\prime}\left|x_{1}-x_{2}\right| \geq 10 \epsilon$ we have

$$
\frac{1}{10} \alpha^{\prime}|x-y| \leq \operatorname{dist}(p(x), p(y)) \text {, }
$$

and

$$
h^{k}\left(f\left([0,1]^{k} \backslash\left(F_{1} \cup \ldots \cup F_{M}\right)\right)\right) \leq c_{1} \alpha^{\prime} .
$$

Using equation (2.1) we get that if $x, y \in F_{i}$ we have

$$
\frac{1}{10} \alpha^{\prime}|x-y|-14 \epsilon \leq \operatorname{dist}(f(x), f(y))
$$

This concludes the proof of Theorem 1.1. 


\section{Curvature estimates}

In this section we prove Lemma 2.1. We first consider the case $k=1$, and then use it to prove the lemma for $k>1$.

\section{Lemma 3.1.}

$$
\sum_{\substack{\mathcal{D}_{0} \cup \mathcal{D}_{1} \\ I \subset[0,1]}} \tilde{\beta}(I)^{2} \operatorname{diam}(I) \lesssim L
$$

This lemma is stated and proved in [9]. The setting we were interested in there was that of Ahlfors-regular curves, however the proof given there for this lemma is correct for the setting we have here. We give most of the proof's details in the appendix of this paper.

We are now ready to prove Lemma 2.1.

Proof of Lemma 2.1. We use Lemma 3.1 and the definition of $\tilde{\beta}^{(k)}$. Fix $i \in\{0,1\}^{k}$ and write

$$
\mathcal{D}^{k}=\mathcal{D}_{i}^{k} \text {. }
$$

We have

$$
\begin{aligned}
& \sum_{\substack{Q \in \mathcal{D}^{k} \\
Q \subset[0,1]^{k}}} \tilde{\beta}^{(k)}(Q)^{2} \operatorname{sd}(Q)^{k} \\
& =\sum_{\substack{Q \in \mathcal{D}^{k} \\
Q \subset[0,1]^{k}}} \int_{g \in G_{k}} \int_{x \in \mathbb{R}^{k} \ominus g \mathbb{R}} \chi_{\{|(x+g \mathbb{R}) \cap 7 Q| \geq \operatorname{sd}(Q)\}} \tilde{\beta}((x+g \mathbb{R}) \cap 7 Q)^{2} \operatorname{sd}(Q) d x d \mu(g) \\
& =\int_{g \in G_{k}} \int_{x \in \mathbb{R}^{k} \ominus g \mathbb{R}} \sum_{\substack{Q \in \mathcal{D}^{k} \\
Q \subset[0,1]^{k}}} \chi_{\{|(x+g \mathbb{R}) \cap 7 Q| \geq \operatorname{sd}(Q)\}} \tilde{\beta}((x+g \mathbb{R}) \cap 7 Q)^{2} \operatorname{sd}(Q) d x d \mu(g) \\
& \lesssim L+\int_{g \in G_{k}} \int_{x \in \mathbb{R}^{k} \in g \mathbb{R}} \chi_{\left\{x \in C[0,1]^{k}\right\}} \sum_{\substack{I \in \mathcal{D}_{0} \cup \mathcal{D}_{1} \\
I \subset[0,1]}} \tilde{\beta}(x+g I)^{2} \operatorname{diam}(I) d x d \mu(g) \\
& \lesssim L+L \int_{g \in G_{k}} \int_{x \in \mathbb{R}^{k} \ominus g \mathbb{R}} \chi_{\left\{x \in C[0,1]^{k}\right\}} d x d \mu(g) \lesssim L+L \int_{g \in G_{k}} d \mu(g) \\
& \lesssim L .
\end{aligned}
$$

where above, the notation $\operatorname{sd}(Q)$ is short for side $(Q)$, the side length of the cube $Q$. 


\section{Appendix}

We review the proof of Lemma 3.1, taken from [9]. For a little more details see the original.

For numbers $r, v \in[0,1]$ we will look at the mapping $\psi^{v, r}:[0,1] \rightarrow[0,1]$ given by $\psi^{v, r}(t)=v+r t \bmod 1$.

For an interval $I \subset[0,1]$ write $I=[a(I), b(I)]$.

Remark 4.1. Let $|I|$ be the diameter of the interval $I$. When doing addition $\bmod 1$, we have (by change of variable) for any $I^{\prime}$ with $\left|I^{\prime}\right|=2^{-k}$

$$
\begin{aligned}
& \sum_{\substack{I \in \mathcal{D}_{0} \\
|I|=2^{-k}}}|I|^{-3} \int_{a(I)}^{b(I)} \int_{x}^{b(I)} \int_{y}^{b(I)} \partial_{1}(x, y, z) d z d y d x \\
& \leq\left|I^{\prime}\right|^{-3} \int_{v=0}^{1} \int_{r=0}^{1} \int_{y \in v+r I^{\prime}} \partial_{1}\left(v+r a\left(I^{\prime}\right), y, v+r b\left(I^{\prime}\right)\right) d y \cdot\left|I^{\prime}\right| d r d v
\end{aligned}
$$

giving

$$
\begin{aligned}
& \sum_{\substack{I \in \mathcal{D}_{0} \\
|I|=2^{-k}}}|I|^{-3} \int_{a(I)}^{b(I)} \int_{x}^{b(I)} \int_{y}^{b(I)} \partial_{1}(x, y, z) d z d y d x \\
& \leq \sum_{\substack{I \in \mathcal{D}_{0} \\
|I|=2^{-k}}}|I|^{-3} \int_{v=0}^{1} \int_{r=0}^{1} \int_{y \in v+r I} \partial_{1}(v+r a(I), y, v+r b(I)) d y \cdot|I| d r|I| d v .
\end{aligned}
$$

Let $I^{\prime}=[a, b] \in \mathcal{D}_{0}$. Define

$$
\partial_{\text {dyadic }}\left(\psi^{v, r}\left(I^{\prime}\right)\right):=\partial_{1}\left((v+r a),\left(v+r \frac{a+b}{2}\right),(v+r b)\right) .
$$

The triangle inequality gives the following lemma.

Lemma 4.2. Let $I \in \mathcal{D}_{0}$. Let $v, r \in[0,1]$ be chosen such that $\psi^{v, r}(I)=$ $[x, z] \ni y$. Then

$$
\partial_{1}(x, y, z) \leq \sum_{\substack{I^{\prime} \in \mathcal{D}, I^{\prime} \subset I \\ y \in \psi^{v, r}\left(I^{\prime}\right)}} \partial_{\text {dyadic }}\left(\psi^{v, r}\left(I^{\prime}\right)\right)
$$

Via telescoping sums, one get the following lemma.

Lemma 4.3. Let $r, v \in[0,1]$ be fixed. Then

$$
\sum_{I^{\prime} \in \mathcal{D}_{0}} \partial_{\text {dyadic }}\left(\psi^{v, r}\left(I^{\prime}\right)\right) \lesssim L
$$


Finally, putting the above together, one gets (with the diameter of $I$ denoted by $|I|)$

$$
\begin{aligned}
& \sum_{I \in \mathcal{D}_{0}}|I|^{-3} \int_{a(I)}^{b(I)} \int_{x}^{b(I)} \int_{y}^{b(I)} \partial_{1}(x, y, z) d z d y d x \\
& \leq \sum_{I \in \mathcal{D}_{0}}|I|^{-3} \int_{v=0}^{1} \int_{r=0}^{1} \int_{y \in v+r I} \partial_{1}(v+r a(I), y, v+r b(I)) d y \cdot|I| d r \cdot|I| d v \\
& \leq \sum_{I \in \mathcal{D}_{0}}|I|^{-3} \int_{v=0}^{1} \int_{r=0}^{1} \sum_{\substack{I^{\prime} \in \mathcal{D}_{0} \\
I^{\prime} \subset I}} \int_{y \in v+r I^{\prime}} \partial_{\text {dyadic }}\left(\psi^{v, r}\left(I^{\prime}\right)\right) \cdot d y \cdot|I| d r \cdot|I| d v \\
& =\sum_{I \in \mathcal{D}_{0}}|I|^{-3} \int_{v=0}^{1} \int_{r=0}^{1} \sum_{\substack{I^{\prime} \in \mathcal{D}_{0} \\
I^{\prime} \subset I}} \partial_{\text {dyadic }}\left(\psi^{v, r}\left(I^{\prime}\right)\right) \cdot r \mathcal{H}^{1}\left(I^{\prime}\right) \cdot|I| d r \cdot|I| d v \\
& =\int_{v=0}^{1} \int_{r=0}^{1} \sum_{I \in \mathcal{D}_{0}} \frac{1}{|I|} \sum_{\substack{I^{\prime} \in \mathcal{D}_{0} \\
I^{\prime} \subset I}} \partial_{\text {dyadic }}\left(\psi^{v, r}\left(I^{\prime}\right)\right) \cdot r \mathcal{H}^{1}\left(I^{\prime}\right) d r d v \\
& =\int_{v=0}^{1} \int_{r=0}^{1} \sum_{I^{\prime} \in \mathcal{D}_{0}} \sum_{I \supset I^{\prime}} \frac{\mathcal{H}^{1}\left(I^{\prime}\right)}{|I|} \partial_{\text {dyadic }}\left(\psi^{v, r}\left(I^{\prime}\right)\right) \cdot r d r d v \\
& \lesssim \int_{v=0}^{1} \int_{r=0}^{1} \sum_{I^{\prime} \in \mathcal{D}_{0}} \partial_{\text {dyadic }}\left(\psi^{v, r}\left(I^{\prime}\right)\right) \cdot r d r d v \lesssim L .
\end{aligned}
$$

which gives Lemma 3.1.

\section{References}

[1] David, G.: Morceaux de graphes lipschitziens et intégrales singulières sur une surface. Rev. Mat. Iberoamericana 4 (1988), no. 1, 73-114.

[2] David, G.: Wavelets and singular integrals on curves and surfaces. Lecture Notes in Mathematics 1465. Springer-Verlag, Berlin, 1991.

[3] David, G. And Semmes, S.: Analysis of and on uniformly rectifiable sets. Mathematical Surveys and Monographs 38. American Mathematical Society, Providence, RI, 1993.

[4] David, G. And Semmes, S.: Fractured fractals and broken dreams. Selfsimilar geometry through metric and measure. Oxford Lecture Series in Mathematics and its Applications 7. The Clarendon Press, Oxford University Press, New York, 1997.

[5] Heinonen, J.: Geometric embeddings of metric spaces. Report. University of Jyväskylä Department of Mathematics and Statistics 90. University of Jyväskylä, Jyväskylä, 2003.

[6] Heinonen, J. and Semmes, S.: Thirty-three yes or no questions about mappings, measures, and metrics. Conform. Geom. Dyn. 1 (1997), 1-12 (electronic). 
[7] Jones, P. W.: Lipschitz and bi-Lipschitz functions. Rev. Mat. Iberoamericana 4 (1988), no. 1, 115-121.

[8] Kirchieim, B.: Rectifiable metric spaces: local structure and regularity of the Hausdorff measure. Proc. Amer. Math. Soc. 121 (1994), no. 1, 113-123.

[9] Schul, R.: Ahlfors-regular curves in metric spaces. Ann. Acad. Sci. Fenn. Math. 32 (2007), no. 2, 437-460.

[10] Schul, R.: Analyst's traveling salesman theorems. A survey. In: In the tradition of Ahlfors and Bers. IV, 209-220. Contemp. Math. 432. Amer. Math. Soc., Providence, RI, 2007.

Recibido: 6 de septiembre de 2007

Revisado: 21 de mayo de 2008

Raanan Schul

UCLA Mathematics Department

Box 951555

Los Angeles, CA 90095-1555, USA

schul@math.ucla.edu

The author is partially supported by NSF DMS 0502747. 